\title{
Ultrasound effects on brain-targeting mannosylated liposomes: in vitro and blood-brain barrier transport investigations
}

This article was published in the following Dove Press journal:

Drug Design, Development and Therapy

24 July 2015

Number of times this article has been viewed

\author{
Ahmed S Zidan ${ }^{1,2}$ \\ Hibah Aldawsari' \\ 'Department of Pharmaceutics \\ and Industrial Pharmacy, Faculty \\ of Pharmacy, King Abdulaziz \\ University, Jeddah, Kingdom of Saudi \\ Arabia; ${ }^{2}$ Department of Pharmaceutics \\ and Industrial Pharmacy, Faculty \\ of Pharmacy, Zagazig University, \\ Zagazig, Egypt
}

\begin{abstract}
Delivering drugs to intracerebral regions can be accomplished by improving the capacity of transport through blood-brain barrier. Using sertraline as model drug for brain targeting, the current study aimed at modifying its liposomal vesicles with mannopyranoside. Box-Behnken design was employed to statistically optimize the ultrasound parameters, namely ultrasound amplitude, time, and temperature, for maximum mannosylation capacity, sertraline entrapment, and surface charge while minimizing vesicular size. Moreover, in vitro blood-brain barrier transport model was established to assess the transendothelial capacity of the optimized mannosylated vesicles. Results showed a dependence of vesicular size, mannosylation capacity, and sertraline entrapment on cavitation and bubble implosion events that were related to ultrasound power amplitude, temperature. However, short ultrasound duration was required to achieve $>90 \%$ mannosylation with nanosized vesicles $(<200 \mathrm{~nm})$ of narrow size distribution. Optimized ultrasound parameters of $65^{\circ} \mathrm{C}, 27 \%$, and 59 seconds for ultrasound temperature, amplitude, and time were elucidated to produce $81.1 \%, 46.6 \mathrm{~nm}$, and $77.6 \%$ sertraline entrapment, vesicular size, and mannosylation capacity, respectively. Moreover, the transendothelial ability was significantly increased by 2.5 -fold by mannosylation through binding with glucose transporters. Hence, mannosylated liposomes processed by ultrasound could be a promising approach for manufacturing and scale-up of brain-targeting liposomes.
\end{abstract}

Keywords: CNS delivery, sizing, lipid based formulations, quality by design, sertraline hydrochloride

\section{Introduction}

The prevalence of central nervous system (CNS) disorders is considered one of the important surfacing areas of unsatisfactory medical need, which represent more hospitalizations, extra long-term attention, and additional chronic suffering than mostly all other diseases. The intensive yet difficult clinical care of these diseases is required due to their influences on the intracerebral functional regions. ${ }^{1}$ These CNS disorders would include but not limited to brain tumors, depression, cerebral ischemic injury, epilepsy, and neurodegenerative disorders. ${ }^{2}$ Two major problems must be overcome to successfully deliver a CNS-acting medication to an intracerebral region, namely permeability and specificity of blood-brain barrier (BBB). BBB would limit the uptake of not only most ionized small molecules but also most large molecules. ${ }^{3,4}$ The specificity to achieve a sufficient concentration of the active medication at a specific intracerebral region is also considered a major challenge. ${ }^{5}$ Therefore, drug delivery systems that can transport BBB then deliver CNS-acting drugs to target specific functional brain regions would propose an alternative therapy of many CNS
Correspondence: Ahmed S Zidan Department of Pharmaceutics and Industrial Pharmacy, Faculty of Pharmacy, King Abdulaziz University, PO Box 80260, Jeddah 21589, Kingdom of Saudi Arabia

Tel +9665 64266682

Fax+966I2695I 696

Email aszidan@kau.edu.sa 
disorders. Different studies in the literature have demonstrated that surface-modified small unilamellar liposomes with $P$-aminophenyl- $\alpha$-D-mannopyranoside, namely mannosylated liposomes, can target the intracerebral region. ${ }^{6,7}$ Mannosylated liposomes can be transported across BBB by glucose transporter 1 (GLUT1), which is mainly expressed by both endothelial cells of the BBB and glioma cells in the brain. ${ }^{8,9}$ Moreover, GLUT1 protein levels are differentially expressed in different intracerebral regions. ${ }^{8}$ Consequently, this different expression of GLUT1 might achieve targeting of mannosylated liposomes in the CNS with a region-specific distribution.

Different techniques are proposed in the literature to prepare multilamellar, large unilamellar, and small unilamellar liposomal vesicles. ${ }^{10,11}$ The application of ultrasound energy to prepare small unilamellar vesicles is widely reported. ${ }^{12,13}$ However, the poor ultrasound experimental set-up might result in poor reproducibility not only to prepare these vesicles but also to achieve the desired targeted delivery. The mechanism of ultrasound energy in liquid media to prepare and comminute these vesicles would be the potentiation of mass transport of their components in a nonhomogeneous mode to reassemble in smaller sizes. ${ }^{14}$ Different studies demonstrated the relationships between the liposomal characteristics and ultrasound power and duration of inputs. ${ }^{15}$ Nevertheless, to increase the robustness, other parameters should also be monitored such as ultrasound field intensity, distance from the ultrasound probe to the vessel's bottom, and the ultrasound wave reflection at the bottom. Rae et al demonstrated that cavitation can be produced in the dispersion media by the ultrasound mechanical waves with the formation of local hot regions and free radicals. ${ }^{16}$ On the other hand, it has been shown that ultrasound path-length would greatly contribute to the resultant temperature rise and free radical formation. ${ }^{17}$ Consequently, these ultrasound parameters, namely energy magnitude, sonication time, ultrasound probe position, and sonication temperature would have a great influence on the ultrasound input applied to the vesicles. Moreover, the effect of the formed hydroxyl radicals on the cavitation bubble collapse is seldom considered in impairment of the produced tensile stresses imparted by ultrasound. ${ }^{17}$

The objective of the current study was to prepare mannosylated liposomes of sertraline hydrochloride, a selective serotonin reuptake inhibitor, as a model CNS-acting medication to enhance its brain delivery. Sertraline has been reported to alleviate depression symptoms such as feelings of intense sadness, helplessness, worthlessness, and impaired functioning. ${ }^{18}$ This work intended to statistically optimize the ultrasound operating parameters using Box-Behnken design to engineer the characteristics of these brain-targeting mannosylated liposomes. Three ultrasound operating parameters, namely sonication amplitude, time, and temperature, were investigated for their influences on liposomal vesicle formation. The formation phase was then related with drug entrapment efficiency (EE), vesicular size, size distribution as expressed by the skewness of size distribution curves, surface charge, and mannosylation capacity. Moreover, based on mouse brain endothelialpolyoma middle T antigen-transformed cell line, in vitro BBB transport model was established to assess the transendothelial capacity of the optimized formulation of mannosylated liposomes.

\section{Materials and methods}

Distearoylphosphosphatidyl ethanolamine was purchased from Avanti Polar Lipids (Alabaster, AL, USA). Sertraline hydrochloride was donated by Riyadh Pharma (Riyadh, KSA). Highly purified polyethylene glycol 200 was purchased from Croda Inc. (Edison, NJ, USA). Chloroform was purchased from PCCA Inc. (Houston, TX, USA). Chromatographic grade phosphoric acids were purchased from VWR Scientific (Bridgeport, NJ, USA). Dicetyl phosphate, $P$-aminophenyl- $\alpha$ D-mannopyranoside, cholesterol, monobasic and dibasic potassium phosphates, and HPLC-grade acetonitrile were purchased from Thermo Fisher Scientific (Waltham, MA, USA).

\section{Preparation of mannosylated liposomes}

Pegylated multilamellar liposomes were prepared using the thin-film method as described by Zidan et al. ${ }^{19}$ Briefly, liposomal suspension was prepared by dissolving phospholipid, cholesterol, dicetyl phosphate, and polyethylene glycol (PEG) 600 in $30 \mathrm{~mL}$ chloroform in a round-bottomed flask at a molar ratio of 40:40:15:5 (mol/mol). Chloroform was evaporated under vacuum using a rotary evaporator (R10 Rotavapor, Buchi, Flawil, Switzerland) at $63^{\circ} \mathrm{C}$ to form a thin film. Evaporation was continued for approximately 15 minutes followed by purging with nitrogen for 5 minutes then overnight vacuum drying at room temperature. Multilamellar liposomes were obtained by film hydration with isotonic sertraline hydrochloride solution in distilled water $(100 \mu \mathrm{g} / \mathrm{mL})$ at $63^{\circ} \mathrm{C}$.

Mannosylation was then performed by conjugating the prepared liposomes with $P$-aminophenyl- $\alpha$-D-mannopyranoside according to a reported method. ${ }^{20}$ Briefly, a specified volume of liposomal suspension, corresponding to $30 \mathrm{mg} / \mathrm{mL}$ of total lipid, was mixed with $7 \mathrm{mg}$ of $P$-aminophenyl$\alpha$-D-mannopyranoside. Excess coupling agent, namely $25 \mathrm{mg}$ 
glutaraldehyde, was then added dropwise to the vesicles followed by incubation at $30^{\circ} \mathrm{C}$ for 30 minutes. In this reaction, the amino groups of phospholipids were coupled with $P$-aminophenyl- $\alpha$-D-mannopyranoside. Unentrapped sertraline hydrochloride, uncoupled $P$-aminophenyl- $\alpha$-Dmannopyranoside, and excess glutaraldehyde were then removed by exhaustive dialyzing against isotonic phosphatebuffered saline. ${ }^{21}$ Mannosylated liposomes were then subjected to size reduction using a probe sonicator (Oscar Ultrasonics Co., Mumbai, Maharashtra, India) at different amplitudes, times, and temperatures according the experimental combinations of Box-Behnken design (Table 1). The free drug and $P$-aminophenyl- $\alpha$-D-mannopyranoside were removed by ultracentrifugation (Beckman XL-90 ultracentrifuge; Beckman, Fullerton, CA, USA) of 65,000 rpm for 2 hours at $4{ }^{\circ} \mathrm{C}$. The resultant liposomal residue was reconstituted with $5 \mathrm{~mL}$ of isotonic solutions and stored at $-2^{\circ} \mathrm{C}$ for further analysis. To evaluate mannosylation capacity, the uncoupled amino groups of the mannosylated liposomes were assessed spectrophotometrically using trinitrobenzene sulfonic acid as a visualization agent. ${ }^{22}$ Mannosylation capacity was then calculated as described in the following equation:

$$
\text { Mannosylation capacity }(\%)=\frac{1-A_{a}}{A_{0}} \times 100,
$$

where $A_{\mathrm{a}}$ is the absorbance of uncoupled amino groups of liposomes after modification with $P$-aminophenyl- $\alpha$-Dmannopyranoside and $A_{0}$ is the absorbance of amino groups of amino groups of liposomes prior to conjugation with $P$-aminophenyl- $\alpha$-D-mannopyranoside.

\section{Entrapment efficiency}

The free sertraline hydrochloride was assessed after ultracentrifugation, Sorvall WX 100+ Ultracentrifuge (Thermo Fisher Scientific) at $90,000 \mathrm{rpm}$ for 90 minutes at $2^{\circ} \mathrm{C}$, using an in-house-developed and validated chromatographic analysis. Quantitation was performed using HPLC Agilent 1200 LC Quaternary series pump coupled with Agilent 1200 high-performance autosampler (Agilent Technologies, Santa Clara, CA, USA). Phenomenex Luna C18 column ( $250 \times 4.6 \mathrm{~mm}, 5 \mu \mathrm{m}$, Phenomenex Co., Torrance, CA, USA) and Zorbax C18 guard column $(12.5 \times 4.6 \mathrm{~mm}, 5 \mu \mathrm{m}$, Agilent Technologies) were used. The mobile phase consisted of $10 \mathrm{mM}$ ammonium formate buffer ( $\mathrm{pH} 4.5$ ) and acetonitrile at $20: 80 \mathrm{v} / \mathrm{v}$ ratio and was pumped isocratically at a flow rate of $1.3 \mathrm{~mL} /$ minute and column temperature of $35^{\circ} \mathrm{C}$. Detector wavelength was fixed at $220 \mathrm{~nm}$ and the injection volume was $20 \mu \mathrm{L}$. The EE of the sertraline hydrochloride in the liposomes after sonication was calculated as the percentage of the initial experimental loading as follows:

$$
\text { Entrapment efficiency }=\frac{\text { Drug }_{\text {total }}-\text { Drug }_{\text {free }}}{\text { Drug }_{\text {total }}} \times 100 \% \text {. }
$$

\section{Particle size and surface charge}

The vesicular size and size distribution, expressed as the skewness value of the size distribution curve, were determined by dynamic light scattering technique. Malvern

\begin{tabular}{|c|c|c|c|c|c|c|c|c|c|}
\hline \multirow[t]{2}{*}{ Batch \# } & \multicolumn{3}{|c|}{ Experimental variables $^{a}$} & \multicolumn{6}{|c|}{ Observed responses $^{b}$} \\
\hline & $\mathrm{XI}\left({ }^{\circ} \mathrm{C}\right)$ & $\mathbf{X} 2(\%)$ & X3(seconds) & $\begin{array}{l}\text { Entrapment } \\
\text { efficiency (\%) }\end{array}$ & $\begin{array}{l}\text { Number-weighted } \\
\text { vesicular size }(\mathrm{nm})\end{array}$ & Skewness & $\begin{array}{l}\text { Zeta potential } \\
(\mathrm{mV})\end{array}$ & Polarity & $\begin{array}{l}\text { Mannosylation } \\
\text { capacity (\%) }\end{array}$ \\
\hline $\mathrm{FI}$ & 35 & 20 & 60 & 93.09 & 5,010 & 0.1 & 0.58 & Positive & 95.3 \\
\hline F2 & 35 & 35 & 30 & 74.61 & 5,975 & 0.03 & 2.18 & Positive & 96.4 \\
\hline F3 & 35 & 35 & 90 & 81.68 & 5,970 & 0.02 & 4.04 & Positive & 97.4 \\
\hline F4 & 35 & 50 & 60 & 87.16 & $2,029.2$ & 0.16 & 0.59 & Negative & 92.6 \\
\hline F5 & 50 & 20 & 30 & 97.55 & 865.3 & 0.49 & 0.55 & Positive & 85.6 \\
\hline F6 & 50 & 20 & 90 & 95.29 & 21.97 & 0.84 & 0.6 & Positive & 54.3 \\
\hline F7 & 50 & 35 & 60 & 85.93 & 600 & 0.2 & 8.6 & Positive & 73.4 \\
\hline F8 & 50 & 35 & 60 & 79.65 & 433.1 & 0.45 & 9.6 & Positive & 80.2 \\
\hline F9 & 50 & 35 & 60 & 83.78 & 29.9 & 0.23 & 8.39 & Positive & 75.2 \\
\hline FIO & 50 & 50 & 30 & 77.04 & 30.2 & 0.98 & 2.02 & Negative & 57.5 \\
\hline FII & 50 & 50 & 90 & 70.24 & 133.7 & 0.46 & 7.97 & Negative & 72.1 \\
\hline $\mathrm{FI} 2$ & 65 & 20 & 60 & 88.08 & 69.1 & 0.65 & 0.57 & Positive & 65.2 \\
\hline $\mathrm{FI3}$ & 65 & 35 & 30 & 72.53 & 961 & 0.98 & 0.61 & Positive & 87.4 \\
\hline $\mathrm{FI} 4$ & 65 & 35 & 90 & 67.59 & 363.5 & 0.99 & 13.29 & Negative & 78.2 \\
\hline FI5 & 65 & 50 & 60 & 65.22 & 164.1 & 0.98 & 0.61 & Positive & 73.4 \\
\hline
\end{tabular}

Table I Experimental variables and the observed response values for sertraline hydrochloride liposomes according to Box-Behnken design

Notes: ${ }^{2}$ Experimental variables include sonication temperature $(X 1)$, amplitude $(X 2)$, and time $(X 3)$. ${ }^{b}$ Standard deviation values for three replicates did not exceed $4 \%$ of the stated response values. 
ALV CGS system (Malvern Instruments, Malvern, UK) with a scattering angle of $90^{\circ}$ at $25^{\circ} \mathrm{C}$. Liposomal suspensions after ultrasound treatment were diluted as required with doubledistilled water before measurement. Electric properties of the produced vesicles, expressed as the resultant zeta potential values, were determined by recording the electrophoretic mobilities using the same Malvern ALV CGS system.

\section{Surface morphology}

The morphology of optimized liposomes was assessed using scanning electron microscopy (JSM-6390 LV; JEOL, Tokyo, Japan) at an accelerated voltage of $15 \mathrm{kV}$. Samples were gold sputter coated before scanning electron microscopy mounting under high vacuum of $45 \mathrm{mT}$ Torr and voltage of $30 \mathrm{mV}$.

\section{In vitro BBB transport model}

Mouse brain endothelialpolyoma middle T antigentransformed cell line (bEnd.3, ATCC ${ }^{\circledR}$ CRL-2299TM, American Type Culture Collection, Manassas, VA, USA) of passage between 5 and 10 were used for sertraline transport experiments. Cells were seeded on $2 \%$ gelatin-coated culture cell inserts at a density of $5.8 \times 10^{4}$ cells per filter and grown for 5 days. The plated cells were then incubated with Dulbecco's Modified Eagle's Medium supplemented with $10 \%$ fetal bovine serum, $2 \mathrm{mmol} / \mathrm{L}$ L-glutamine, $100 \mu \mathrm{g} / \mathrm{mL}$ endothelial cell growth factor, $100 \mathrm{U} / \mathrm{mL}$ penicillin, and $100 \mu \mathrm{g} / \mathrm{mL}$ streptomycin. Cells were maintained at $5 \% \mathrm{CO}_{2}$, $95 \%$ relative humidity, and $37^{\circ} \mathrm{C}$. The cells were allowed to adhere for 24 hours, and the media was replaced every other day until the cells reached $\sim 80 \%$ confluency. The cells were then subcultured on 12 transwell inserts for unidirectional transport experiment of the optimized formulation. To test the integrity of the monolayer, cells' tightness was assessed before and after the transport study by measuring the transendothelial electrical resistance (TEER) with a Millicell ERS-2 Voltohmmeter (EMD Millipore, Billerica, MA, USA). After appropriate treatment, $1.25 \mathrm{mg} / \mathrm{mL}$ of the optimized formulation was added into the apical side of the BBB model. At 2-, 4-, 8-, 12-, and 16-hour intervals, aliquots were withdrawn from the basolateral side for sertraline assay using the chromatographic analysis method after appropriate dilution. Triplicate samples were performed. The accumulated permeating sertraline amount was calculated by dividing the accumulated amount of the drug recovered in the basolateral side by its initial concentration in the apical side.

For transport inhibition study, either $0.07 \mathrm{M} / \mathrm{mL}$ cytochala$\sin \mathrm{B}$ or $50 \mu \mathrm{M} / \mathrm{mL}$ phenobarbital as transport inhibitors were added to the apical chamber and incubated with the bEnd.3 cells at $37^{\circ} \mathrm{C}$ for 30 minutes. Following this preincubation with the inhibitors, the permeation study was performed the same as described earlier.

\section{Results and discussion}

The treatment of CNS disorders intensely depends on the transport of CNS-acting medications into the brain. In a previous study, liposomal surface was modified with $P$-aminophenyl- $\alpha$-D-mannopyranoside to investigate its specific interaction with GLUT1, hence facilitating the transport of medications across the BBB. ${ }^{20}$ Nevertheless, GLUT1 is widely distributed in the CNS and might contribute significantly to liposomal distribution in brain. ${ }^{23}$ The aim of the current study was to propose mannosylated liposomes of sertraline hydrochloride through systematic methodology. Moreover, ultrasound effects on the critical liposomal characteristics was also investigated. Pegylation was performed to improve circulation of the liposomes through minimizing the termination by reticuloendothelial system. ${ }^{24}$ It has been reported that vesicular sizes of $>200 \mathrm{~nm}$ are exposed to splenic filtration, because they cannot pass through the interendothelial cell slits in the walls of venous sinuses. ${ }^{25}$ Because the vesicular size affects liposomal endocytosis by brain capillary endothelial cells, liposomes are developed with vesicular size $>200 \mathrm{~nm}$ in diameter. ${ }^{26}$ In the current investigation, an optimization study based on Box-Behnken design was constructed to optimize ultrasound parameters, namely sonication amplitude, time, and temperature, regarding drug EE, vesicular size, size distribution, surface charge, and mannosylation capacity.

\section{Influence of ultrasound variables on vesicular size and size distribution}

To explore the influence of ultrasound parameters on the resultant liposomal size and extent of size distribution expressed by the resultant skewness value of size distribution curves, the liposomal size was recorded using number-weighing strategy. Number-weighted vesicular size is the same as measuring vesicle's size using a microscope. It assigns a size value to each vesicle inspected so that it builds a number distribution wherein each vesicle has equal weighting once the final distribution is calculated. In multiple light scattering techniques, the scattered intensity of the light during size measurement is weighted by both the liposomal mass and the other vesicular factors such as the hydrodynamic vesicular radius. Numberweighted measurement is independent of the scattering angle, and hence, it would describe the obtained results better than the intensity and volume weightings. 
Table 2 Results of regression analysis to test the design in portions

\begin{tabular}{|c|c|c|c|c|c|c|c|c|c|c|}
\hline \multirow[t]{2}{*}{ Term } & \multicolumn{2}{|c|}{$\begin{array}{l}\text { Entrapment } \\
\text { efficiency (\%) }\end{array}$} & \multicolumn{2}{|c|}{$\begin{array}{l}\text { Number-weighted } \\
\text { vesicular size }(\mathrm{nm})\end{array}$} & \multicolumn{2}{|l|}{ Skewness } & \multicolumn{2}{|c|}{ Zeta potential $(\mathrm{mV})$} & \multicolumn{2}{|c|}{$\begin{array}{l}\text { Mannosylation } \\
\text { capacity (\%) }\end{array}$} \\
\hline & Estimate & $P$-value & Estimate & $P$-value & Estimate & $P$-value & Estimate & $P$-value & Estimate & $P$-value \\
\hline Intercept & 83.12 & 0.0001 & 354.33 & 0.4313 & 0.293 & 0.0128 & 8.86 & 0.0012 & 76.27 & 0.0001 \\
\hline $\mathrm{XI}\left({ }^{\circ} \mathrm{C}\right)$ & -5.39 & 0.0143 & $-2,177.6$ & 0.0004 & 0.413 & 0.0003 & 0.96 & 0.2944 & -9.69 & 0.0026 \\
\hline X2 (\%) & -9.29 & 0.0014 & -451.14 & 0.1354 & 0.064 & 0.2369 & I.II & 0.2339 & -0.60 & 0.7439 \\
\hline X3 (seconds) & -0.86 & 0.5797 & -167.16 & 0.539 & 0.021 & 0.6731 & 2.56 & 0.026 & -3.11 & 0.1332 \\
\hline$\times 1 * \times 2$ & -4.23 & 0.0963 & -768.94 & 0.0349 & 0.068 & 0.3607 & 0.01 & 0.9951 & 2.73 & 0.3178 \\
\hline$\times 1 * \times 3$ & -3.05 & 0.2066 & -149.37 & 0.6943 & 0.003 & 0.9717 & 2.71 & 0.0472 & -2.55 & 0.3469 \\
\hline$\times 2 * \times 3$ & -1.13 & $0.607 \mid$ & -236.7 I & 0.5385 & -0.220 & 0.0220 & 1.47 & 0.2599 & -11.48 & 0.0055 \\
\hline$X I * X I$ & -5.33 & 0.0463 & $-2,258.5$ & 0.0018 & -0.007 & 0.9277 & 3.01 & 0.0448 & -13.92 & 0.0028 \\
\hline$\times 2 * \times 2$ & -5.59 & 0.0484 & -794.77 & 0.0865 & 0.186 & 0.0449 & 5.26 & 0.0073 & -8.56 & 0.0204 \\
\hline$\times 3 * \times 3$ & -3.68 & 0.1477 & -703.23 & 0.1183 & 0.216 & 0.0272 & -0.81 & 0.5286 & -0.33 & 0.9014 \\
\hline$R^{2}$ & 0.9396 & & 0.9625 & & 0.9546 & & 0.8973 & & 0.9535 & \\
\hline RMSE & 4.14 & & 717.33 & & 0.13 & & 2.32 & & 4.91 & \\
\hline
\end{tabular}

Notes: Experimental variables include sonication temperature $(\mathrm{X} 1)$, amplitude $(\mathrm{X} 2)$, and time $(\mathrm{X} 3)$, whereas $R^{2}$ and RMSE values are coefficients of multiple determination for predicted versus measured values and RMSE values for models' predictabilities, respectively. Bold figures demonstrate significant variables $(P<0.05)$ on the investigated responses.

Abbreviation: RMSE, root mean square error.

The obtained results demonstrated a potential of ultrasound input to comminute the vesicles to nanosize range with a homogenous size distribution for most formulations. For the various ultrasound parameter combinations, Table 1 shows that the number-weighted vesicular size ranged from $21.97 \mathrm{~nm}$ (F6) with a skewness value of $0.84-5975 \mathrm{~nm}$ (F2) with a skewness value of 0.02 . On the other hand, maximum and minimum skewness values, of 0.99 (F14) for a vesicular size of $363.5 \mathrm{~nm}$ and 0.02 (F3) for a vesicular size of 5,970 nm, respectively, were noted. The "Estimate" column in Table 2 demonstrates each factor's relative strength to the other factors. In particular, the higher the estimate value of the factor, the more the influence of that factor on the response. Moreover, an estimate with positive sign shows a direct relationship of the factor with the response, whereas a negative estimate shows an inverse relationship. The most significant factors affecting the number-weighted vesicular size were the individual and polynomial effects of the sonication temperature and the interaction term of sonication temperature and amplitude $(P<0.05$, Table 2 and Figure 1). The obtained results showed that increasing the sonication temperature resulted in a linear and polynomial decrease in vesicles size, and the individual term (X1) had a more dominant influence (Figure 1). On the other

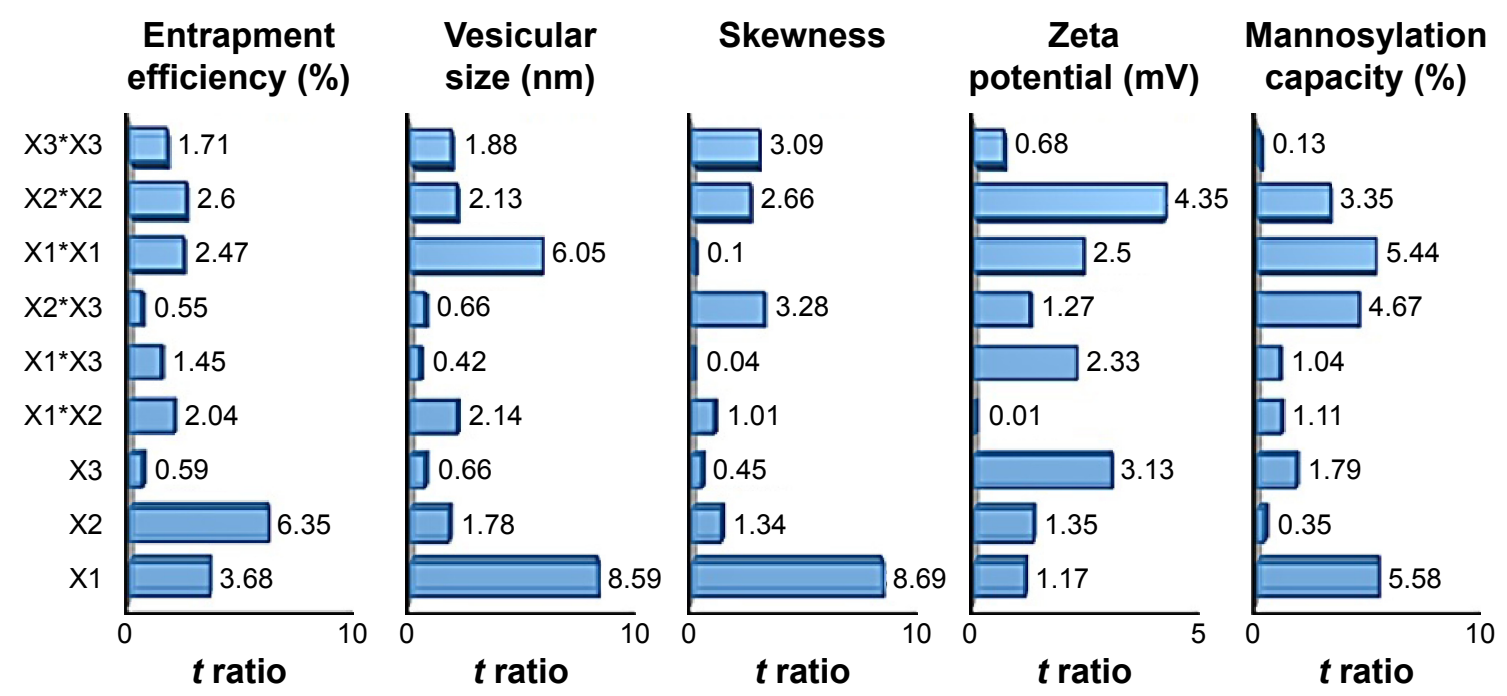

Figure I Pareto charts of standardized effects of various factors, namely sonication temperature (XI), amplitude (X2), and time (X3), on the investigated responses. 
hand, increasing the magnitude of interaction between the sonication temperature and amplitude was associated with a less significant decrease in the vesicular size (Figure 1). Regarding the size distribution, skewness values increased significantly by increasing not only sonication temperature but also polynomial term of sonication amplitude and time (Table 2 and Figure 1). The models' predictabilities for the vesicular size and skewness values were acceptable $(P<0.05)$ with root mean square error values of 717.33 and 0.13 , respectively. Moreover, regression coefficients $\left(R^{2}\right)$ of 0.9625 and 0.9546 were obtained for plotting the predicted number-weighed vesicular size and skewness values versus their experimental values, respectively (Table 2 and Figure 2). On the other hand, plotting the models' residuals against the actual values did not show any trend to indicate both nonsignificant models' errors and models robustnesses. After neglecting the insignificant variables, the reduced linear model equations that explain the effect of only the significant variables on vesicular size and skewness can be expressed as:

$$
\begin{aligned}
& 354.3-2,177.6 \times\left[\frac{\left[\mathrm{X} 1\left({ }^{\circ} \mathrm{C}\right)-50\right]}{15}\right] \\
& \text { Number } \\
& \text { weighed } \\
& \text { vesicular } \\
& \text { size (nm) } \\
& = \\
& -\left[\frac{\left[\mathrm{X} 1\left({ }^{\circ} \mathrm{C}\right)-50\right]}{15}\right] \times\left[\left[\frac{[\mathrm{X} 2(\%)-50]}{15}\right]\right] \\
& \times\left[\left[\frac{[\mathrm{X} 2(\%)-35]}{15}\right] \times 768.94\right] \\
& -\left[\frac{\left[\mathrm{X} 1\left({ }^{\circ} \mathrm{C}\right)-50\right]}{15}\right] \times\left[\left[\frac{\mathrm{X} 2(\%)-35]}{15}\right] \times 2,258.5\right] \\
& 0.29+0.4125 \times\left[\frac{\left[\mathrm{X} 1\left({ }^{\circ} \mathrm{C}\right)-50\right]}{15}\right]+\left[\frac{[\mathrm{X} 2(\%)-35]}{15}\right] \\
& \text { Skewness }=\times\left[\left[\frac{[\mathrm{X} 3 \text { (seconds) }-60]}{30}\right] \times 0.22\right]+\left[\frac{[\mathrm{X} 2(\%)-35]}{15}\right] \\
& \times\left[\left[\frac{[\mathrm{X} 2(\%)-35]}{15}\right] \times 0.18\right]+\left[\frac{[\mathrm{X} 3(\text { seconds })-60]}{30}\right] \\
& \times\left[\left[\frac{[\mathrm{X} 3 \text { (seconds })-60]}{30}\right] \times 0.2\right]
\end{aligned}
$$

Before applying ultrasound energy, all formulations were large multilamellar vesicles with wide size distributions. Most vesicles were more than $5 \mu \mathrm{m}$ in size with approximately $5 \%$ of vesicles below $200 \mathrm{~nm}$. The negative effect of sonication temperature on the resultant size would be explained by the cavitation and bubble implosion which contributed to the heat energy of the dispersion. ${ }^{12}$ These effects would result in vesicular friction within the bulk dispersion from the mixing effect and vesicular friction between the bulk dispersion with the stationary boundary layer adjacent to the side of the vessel. These augmented friction forces by temperature increase could be responsible for sustaining effective cavitation to reduce the vesicular size. ${ }^{17}$ On the other hand, a decrease of the vesicular size with homogenous distribution was observed with the increase of sonication amplitude (Table 2 and Figure 3 ). This effect could be described by increasing the sonication power to exert greater shear forces within the dispersion. The greater extent of streaming at higher ultrasound amplitude stimulated higher mixing of the liposomal dispersion and hence more homogeneity. ${ }^{12}$ Different hypotheses in the literature suggested that ultrasound energy would randomly comminute large multilamellar vesicles to unilamellar vesicles that fold up into thermodynamically stable vesicles with narrow size distribution. ${ }^{27}$ Alternatively, fine unstable vesicles formed in microdomains of ultrasound probe might fuse together to form slightly larger, however stable, vesicles.

\section{Influence of ultrasound variables on zeta potential}

Considering the electrical double-layer theory, assessing the surface charge at the vesicular surface by electrophoresis has been performed by many researchers. ${ }^{28}$ Hence, assessment of zeta potential could provide information about the charge intensity outside the hydrodynamically stagnant layer in relation to counter charges inside of the stagnant layer. For the different ultrasound variable combinations, Table 1 shows that zeta potential values varied from $0.55 \mathrm{mV}$ (F5) at the positive side to $13.29 \mathrm{mV}$ (F14) at the negative side. The most significant factors affecting the electrical properties of the liposomal suspension were the polynomial term of sonication amplitude followed by sonication time then sonication temperature $(P<0.05$, Table 2 and Figure 1). The obtained results showed that increasing the significant sonication parameters resulted in significant increase in surface charge intensity (Figures 1 and 3). It is worth noting that adsorbed conjugated acidic moieties of sertraline at the surface of the neutral liposomes would impart positive electrophoretic mobilities. However, the resultant positive zeta potential decreased by the negatively charged counter ions of dicetyl phosphate and PEG to change the sign to negative. This result would indicate the effective coating of the vesicular surface by PEG and dicetyl phosphate with lesser association with sertraline moieties. The positive influence of sonication parameters on the electrophoretic 

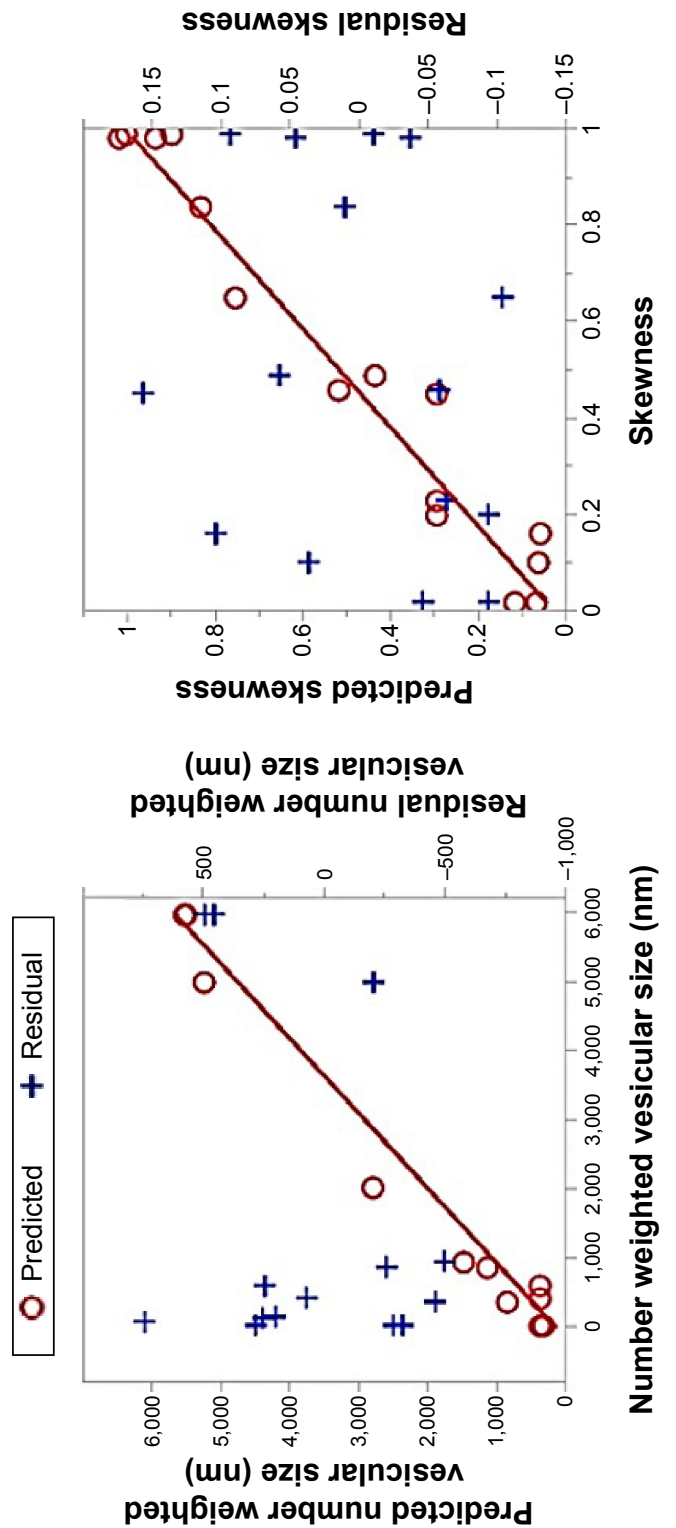

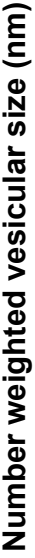
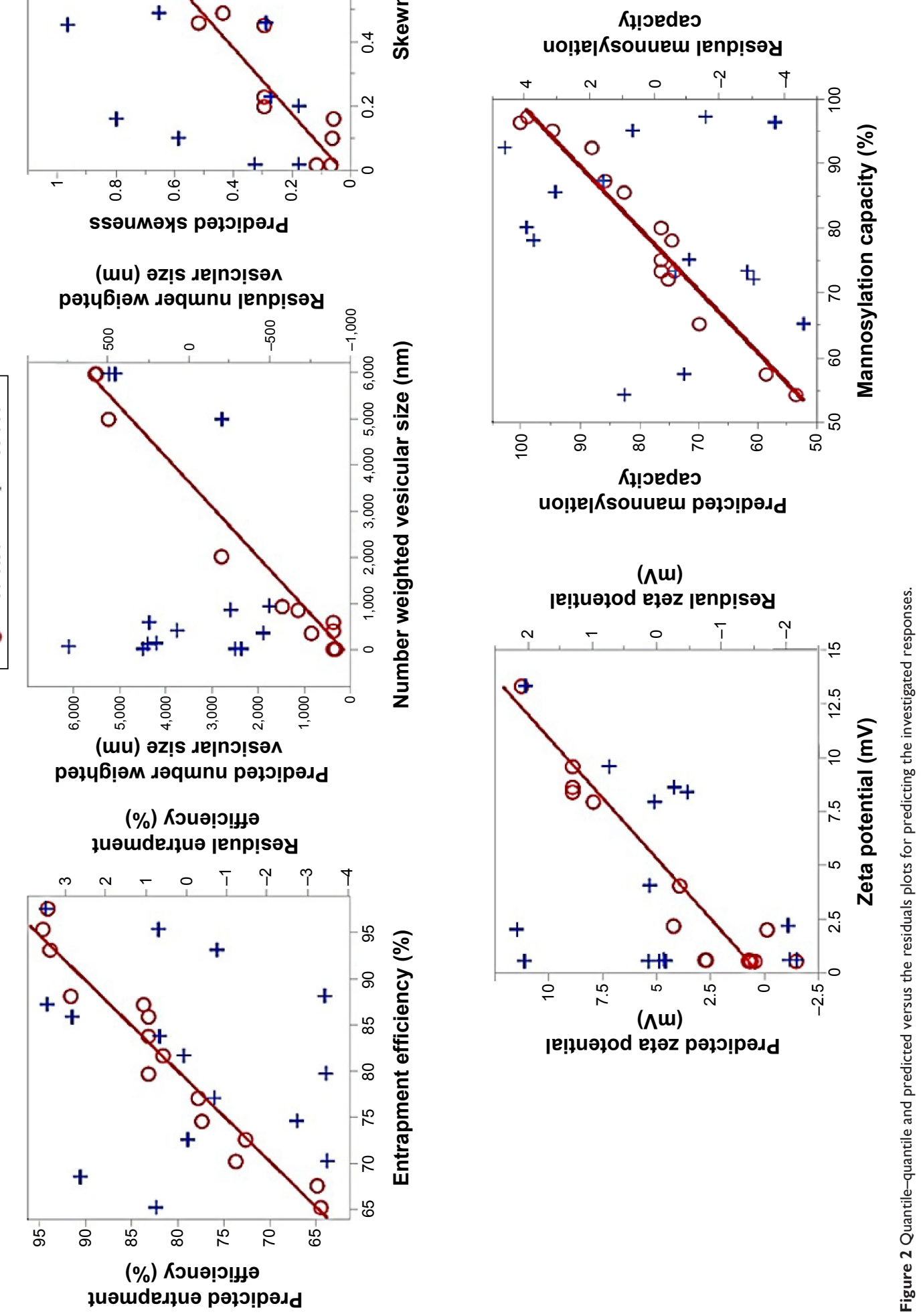

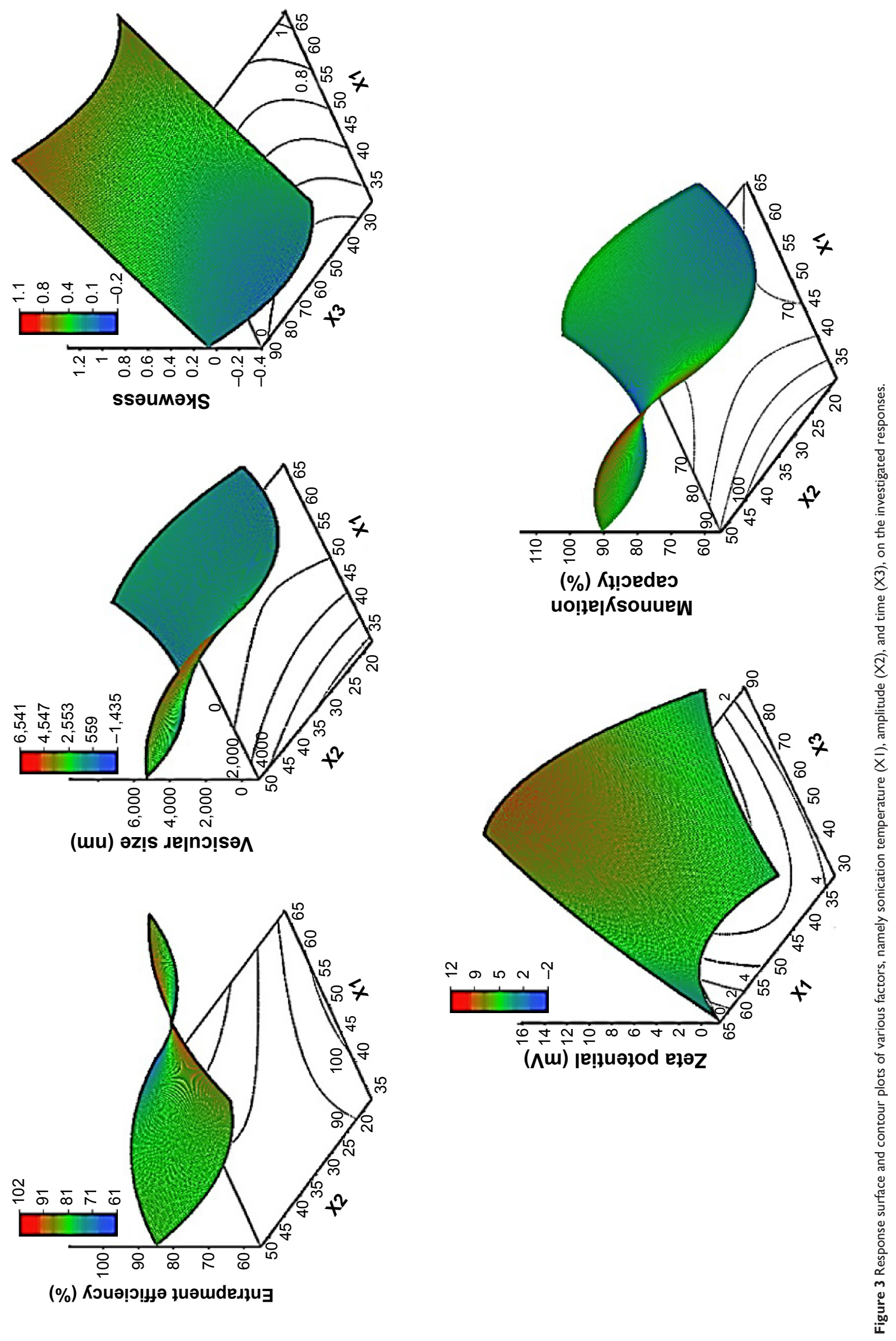
mobilities could be explained by their influences on the resultant vesicular size. This behavior could be explained by the shift of hydrodynamic slipping plane toward the liposomal surface as the vesicular size decreases. In particular, as the vesicular size decreased, the head groups of the lipids became closely packed with each other so that the electric repulsion between the charged groups is increased to stabilize the formed vesicles. ${ }^{29}$ Thus, constraints should be applied to ultrasound parameters to not only produce the desired vesicular size but also maintain the required entropy at the vesicular surface for sufficient repulsive force. The constructed model showed good predictability of $P=0.0483$ for zeta potential estimation with quantile-quantile correlation coefficients of 0.8973 and root mean square error value of 2.32 (Table 2 and Figure 2). The linear reduced model equation to predict surface charge of the liposomal dispersion can be expressed as:

$$
\begin{aligned}
& 8.86+2.5675 \times\left[\frac{[\mathrm{X} 3 \text { (seconds) }-60]}{30}\right]+\left[\frac{\left[\mathrm{X} 1\left({ }^{\circ} \mathrm{C}\right)-50\right]}{15}\right] \\
\underset{\text { potential }}{\text { Zeta }}= & \times\left[\left[\frac{[\mathrm{X} 3 \text { (seconds) }-60]}{30}\right] \times 2.705\right]+\left[\frac{\left[\mathrm{X} 1\left({ }^{\circ} \mathrm{C}\right)-50\right]}{15}\right] \\
& *\left[\left[\frac{\left[\mathrm{X} 1\left({ }^{\circ} \mathrm{C}\right)-50\right]}{15}\right] \times 3.01\right]+\left[\frac{[\mathrm{X} 2(\%)-35]}{15}\right] \\
& \times\left[\left[\frac{[\mathrm{X} 2(\%)-35]}{15}\right] \times 5.2\right]
\end{aligned}
$$

\section{Influence of ultrasound variables on drug entrapment}

The association of dicetyl phosphate with the lipid matrix would impart negatively charged environment for electrostatic interaction with sertraline. Most probably, the insertion of sertraline into phospholipid vesicles occurs due to both hydrophobic interactions of the weak base form and association of its conjugated acid with negatively charged bilayer structures. ${ }^{30}$ Table 1 shows that the EE varied from $65.22 \%$ (F15) to $97.55 \%$ (F5) by changing the combinations of ultrasound parameters. The most significant factors were individual and polynomial terms of both sonication temperature and amplitude $(P<0.05$, Table 2 and Figure 1). The obtained results showed that increasing all sonication parameters would contribute to a decrease in the EE and sonication amplitude had a more predominant effect (Figure 1). The results would suggest that ultrasound's shear forces to disrupt the multilamellar vesicles formed into unilamellar vesicles caused significant sertraline leakage from liposomes. ${ }^{31}$ Another possible explanation would be that the limited aqueous compartment for sertraline entrapment existed as unilamellar vesicles were assembled during sonication. ${ }^{32}$ Moreover, the negative effect of thermal ultrasound on sertraline entrapment could be attributed to the acoustic cavitation-derived shear force to facilitate drug leakage. In particular, the cavitationderived shear force could improve the permeability of vesicular lamellae by generating transient pore formation in the lipid membranes of liposomes. ${ }^{33}$ On the other hand, sertraline leakage at the high temperature of sonication would be a consequence of the enhanced phospholipid molecular mobility to partition the hydrophilic drug with the dispersion medium. ${ }^{30}$

The prediction confidence level of the model to sertraline entrapment was $89.3 \%$ and an acceptable correlation was obtained between the observed and predicted entrapment percentages as indicated by the $R^{2}$ value of 0.9396 with a root mean square error of 4.14 (Table 2 and Figure 2). The linear model equation to predict sertraline entrapment can be expressed as:

$$
\begin{aligned}
& 83.12-5.39 \times\left[\frac{\left[\mathrm{X} 1\left({ }^{\circ} \mathrm{C}\right)-50\right]}{15}\right]-9.29345 \\
& +\left[\frac{[\mathrm{X} 2(\%)-35]}{15}\right]+\left[\frac{\left[\mathrm{X} 1\left({ }^{\circ} \mathrm{C}\right)-50\right]}{15}\right] \\
\text { Entrapment } & = \\
\text { efficiency }(\%) & \times\left[\left[\frac{\left.\mathrm{XX}\left({ }^{\circ} \mathrm{C}\right)-50\right]}{15}\right] \times-5.33\right]+\left[\frac{[\mathrm{X} 2(\%)-35]}{15}\right] \\
& \times\left[\left[\frac{[\mathrm{X} 2(\%)-35]}{15}\right] \times-5.5975\right]
\end{aligned}
$$

\section{Influence of ultrasound variables on mannosylation capacity}

The conjugation capacity between mannopyranoside moieties and vesicular surface would significantly affect the targeting ability and cellular uptake of the vectors. In particular, the length and flexibility of the linking agent between the saccharide head group and vesicular surface mainly influence the target specificity and uptake of liposome by macrophages. ${ }^{34}$ In this regard, liposomes modified with a higher content of saccharide residues would exhibit more efficient cellular recognition and internalization rather than vesicles with lower sugar association. Consequently, theoretically, the more $P$-aminophenyl- $\alpha$-D-mannopyranoside loaded onto the vesicles, the better the targeted delivery could be attained. 
However, excessive mannosylation could initiate the disintegration of liposomes in the circulation due to drug leakage out of the vector and cause a remarkable decline of the drugloading capacity. ${ }^{7}$ For this reason, a constraint should be applied to optimize mannosylation capacity in order to not only improve the targeting potential but also achieve acceptable drug entrapment.

In the current study, the mannosylation capacity percentages were fluctuating from $54.3 \%$ (F6) to $97.4 \%$ (F3) by varying the ultrasound parameters under investigation (Table 2). As shown in Figure 1 and Table 2, individual and polynomial terms of sonication temperature had the most significant effects $(P<0.05)$ to lower the mannosylation capacity followed by the interaction term of sonication amplitude and time. With an increase in the mannosylation capacity, the loading percent of sertraline showed no specific trend at constant dicetyl phosphate loading (Figure 4). This result would confirm two facts. First, mannopyranoside moieties were associated at the vesicular surface with minimal effect on both lamellar structure and volume of aqueous compartment. Second, sertraline entrapment was mostly affected by its interaction with the lipid pool with no influence of coupling process. On the other hand, a significant increase in mannosylation capacity was observed by increasing vesicular size through a logarithmic relationship (Figure 4). In particular, mannosylation capacity would be predicted from vesicular size using the following equation:

$$
\begin{gathered}
\text { Mannosylation } \\
\text { capacity }(\%)
\end{gathered}=6.61 \times \text { vesicular size }(n m)+39.44
$$

This relationship would describe the negative influence of sonication parameters on the resultant mannosylation capacity. After the coupling reaction, the uncoupled $P$-aminophenyl- $\alpha$-D-mannopyranoside and the coupling agent were removed by the exhaustive dialysis. Hence, the production of smaller vesicles would create free lipid surfaces devoid of mannopyranoside to lower the overall mannosylation capacity. On the other hand, at lower values of sonication parameters, larger multilamellar vesicles were produced with mannosylated surfaces. Therefore, 1:4 w/w was deemed effective as the mannopyranoside moieties to lipid matrix for surface modification with sertraline entrapment of approximately $90 \%$ and vesicular size below $200 \mathrm{~nm}$. Similar results were observed by other researchers for mannosylation of polyethylene glycol-coated phosphatidylethanolamine liposomes to entrap $90 \%$ green fluorescence protein plasmid as a model gene at vesicular size of $237 \mathrm{~nm}$ and ligand to carrier ratio of $10 \% .^{7}$ The developed prediction model to predict mannosylation capacity as a function of sonication parameters showed good predictability of $P=0.0077$ with quantile-quantile correlation coefficient of 0.9535 and root mean square error of $4.91 \%$ (Table 2 and Figure 2). The linear reduced model equation to predict mannosylation capacity is given below:

$$
76.2-9.6875 \times\left[\frac{\left[\mathrm{X} 1\left({ }^{\circ} \mathrm{C}\right)-50\right]}{15}\right]
$$

$\begin{aligned} \begin{array}{l}\text { Mannosy- } \\ \text { lation }\end{array} & -\left[\frac{[\mathrm{X} 2(\%)-35]}{15}\right] \times\left[\left[\frac{[\mathrm{X} 3(\text { seconds })-60]}{30}\right] \times 11.475\right] \\ \begin{array}{l}\text { capacity } \\ (\%)\end{array} & +\left[\frac{\left[\mathrm{X} 1\left({ }^{\circ} \mathrm{C}\right)-50\right]}{15}\right] \times\left[\left[\frac{\left[\mathrm{X} 1\left({ }^{\circ} \mathrm{C}\right)-50\right]}{30}\right] \times-13.9\right] \\ & +\left[\frac{[\mathrm{X} 2(\%)-35]}{15}\right] \times\left[\left[\frac{\mathrm{X} 2(\%)-35]}{15}\right] \times-8.5\right] .\end{aligned}$

\section{Model validation and analysis of variance}

The developed predication equations revealed linear relationships between the experimental and predicted values for all responses with $R^{2}$ values $>0.89$ and root mean square errors $<5 \%$ (Table 2 and Figure 2). The individual residuals were scattered about zero with no trends to not only exclude any missing values, nonconstant variance, or outliers but also evidence normal distribution of the experimental data (Figure 2). In addition, significant difference existed between Cook's distance values for all individual responses and the conforming threshold values to confirm the absence of outliers or abnormalities. At $P<0.05$ and degree of freedoms of 9 and 5 for the models' predictions and errors, respectively, sum of squares values of $1,332.5,66 \times 10^{6}, 1.89,235.6$, and $2,474.9$ with mean of squares values of $148.5,7.3 \times 10^{6}, 0.21$, 26.1, and 274.9 were obtained for EE, number-weighted vesicular size, skewness, zeta potential, and mannosylation capacity, respectively (Table 3 ). Values of $F$-ratio, Prob $>F$, and lack of fit $F$-ratio (FLOF) were used to evaluate the predictability of the constructed models. Greater than their corresponding critical values, $F$-ratios of $8.6,14.7,11.6,4.8$, and 11.34 and Prob $>F$-values as low as $0.0143,0.0046$, $0.0073,0.0483$, and 0.0077 for EE, number-weighted vesicular size, skewness, zeta potential, and mannosylation capacity, respectively, were obtained (Table 3). Moreover, FLOF values were more than 0.05 to evidence the nonsignificance of models' lack of fit for all responses. Therefore, analysis of variance results demonstrated the precision and accuracy of the developed prediction models. 
A

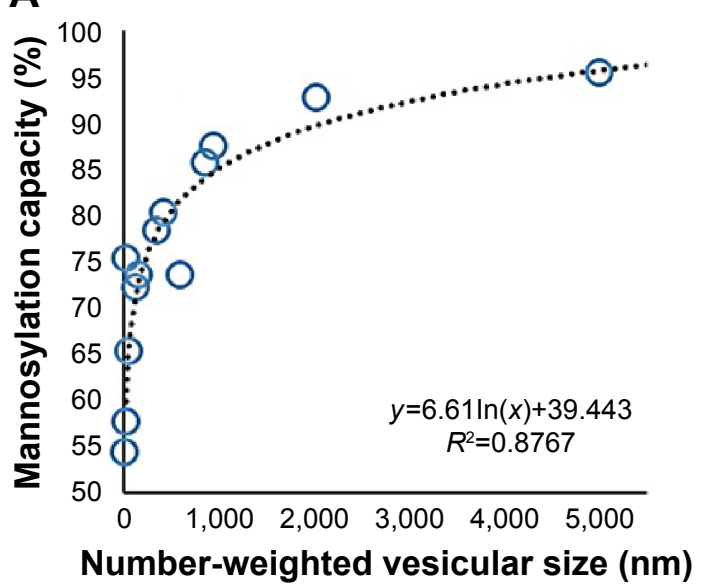

B

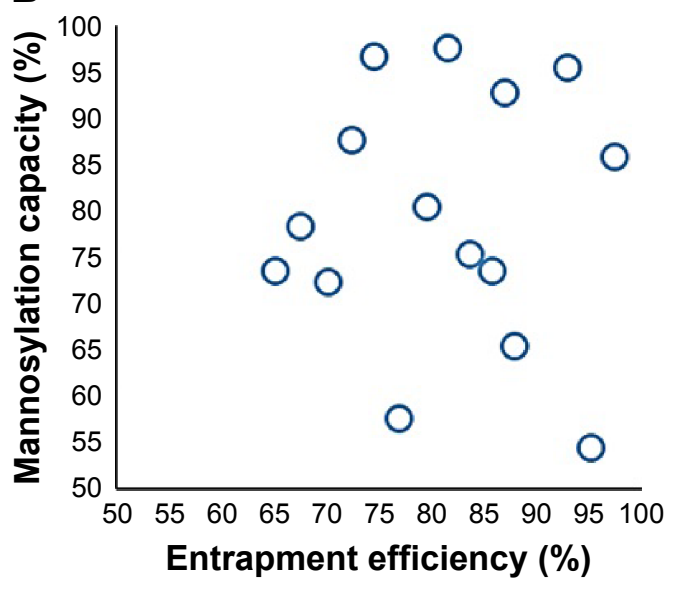

Figure 4 Mannosylation capacity percentages of sertraline liposomes processed with different sonication parameters as the function of either number-weighted vesicular size $(\mathbf{A})$ or drug entrapment efficiency values $(\mathbf{B})$.

Note: Standard deviation values for three replicates did not exceed $4 \%$ of the stated responses' values.

\section{Optimization and desirability function}

A generalized desirability function was employed to maximize EE, skewness, zeta potential, and mannosylation capacity while minimizing the vesicular size below $200 \mathrm{~nm}$. Optimized ultrasound parameters were obtained with predicted responses of $81.1 \%, 46.6 \mathrm{~nm}, 0.68,12.3 \mathrm{mV}$, and $77.6 \%$ for EE, vesicular size, skewness, zeta potential, and mannosylation capacity, respectively, at ultrasound temperature, amplitude, and time of $65^{\circ} \mathrm{C}, 27 \%$, and 59 seconds, respectively. To validate the optimization process, a fresh formulation was processed with these optimized ultrasound parameters that resulted in EE, vesicular size, skewness, zeta potential, and mannosylation capacity
$80 \%, 55 \mathrm{~nm}, 0.75,11.8 \mathrm{mV}$, and $75 \%$, respectively. At $P<0.05$, nonsignificant difference was observed between the predicted and measured values to demonstrate the feasibility of the optimization process to understand the influence of ultrasound to prepare mannosylated liposomes for brain targeting.

The scanning electron microscopy image of the optimized formulation shows the spherical nature of intact liposomes with approximated vesicular size range of $180 \mathrm{~nm}$ and narrow size distribution (Figure 5). These observations were in a good agreement with those obtained by light scattering that unveiled a vesicular size of approximately $50 \mathrm{~nm}$ and skewness value of 0.6.

Table 3 Summary of ANOVA testing for evaluating the significance of the model in portions

\begin{tabular}{|c|c|c|c|c|c|c|c|}
\hline \multirow[t]{2}{*}{ Responses } & \multicolumn{5}{|c|}{ ANOVA parameters } & \multicolumn{2}{|c|}{ Lack-of-fit parameters } \\
\hline & DF & SS & MS & $F$ ratio & Prob $>F$ & $F$ ratio & Prob $>F$ \\
\hline \multicolumn{8}{|c|}{ Entrapment efficiency (\%) } \\
\hline Model & 9 & $1,332.5$ & 148.5 & 8.6 & 0.0143 & 2.3 & 0.3344 \\
\hline Error & 5 & 85.7 & 17.4 & & & & \\
\hline \multicolumn{8}{|c|}{ Number-weighted vesicular size $(\mathrm{nm})$} \\
\hline Model & 9 & $66 \times 10^{6}$ & $7.3 \times 10^{6}$ & 14.7 & 0.0046 & 9.3 & 0.0985 \\
\hline Error & 5 & $2.5 \times 10^{6}$ & $0.51 \times 10^{6}$ & & & & \\
\hline \multicolumn{8}{|l|}{ Skewness } \\
\hline Model & 9 & 1.89 & 0.21 & 11.6 & 0.0073 & 0.945 & 0.5511 \\
\hline Error & 5 & 0.09 & 0.018 & & & & \\
\hline \multicolumn{8}{|c|}{ Zeta potential (mV) } \\
\hline Model & 9 & 235.6 & 26.1 & 4.8 & 0.0483 & 20.8 & 0.0861 \\
\hline Error & 5 & 26.9 & 5.3 & & & & \\
\hline \multicolumn{8}{|c|}{ Mannosylation capacity (\%) } \\
\hline Model & 9 & $2,474.9$ & 274.96 & 11.34 & 0.0077 & 2.57 & 0.2921 \\
\hline Error & 5 & |20.7| & 24.43 & & & & \\
\hline
\end{tabular}

Abbreviations: ANOVA, analysis of variance; DF, degree of freedom; SS, sum of squares; MS, mean of square values. 
A

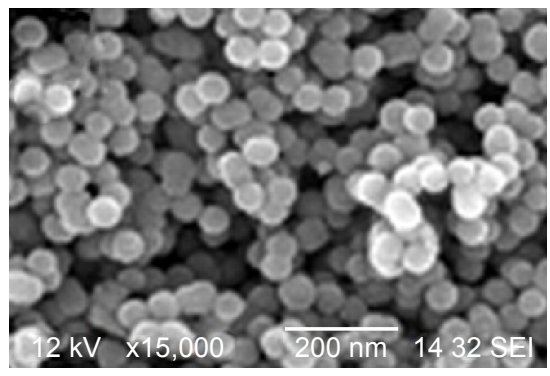

B

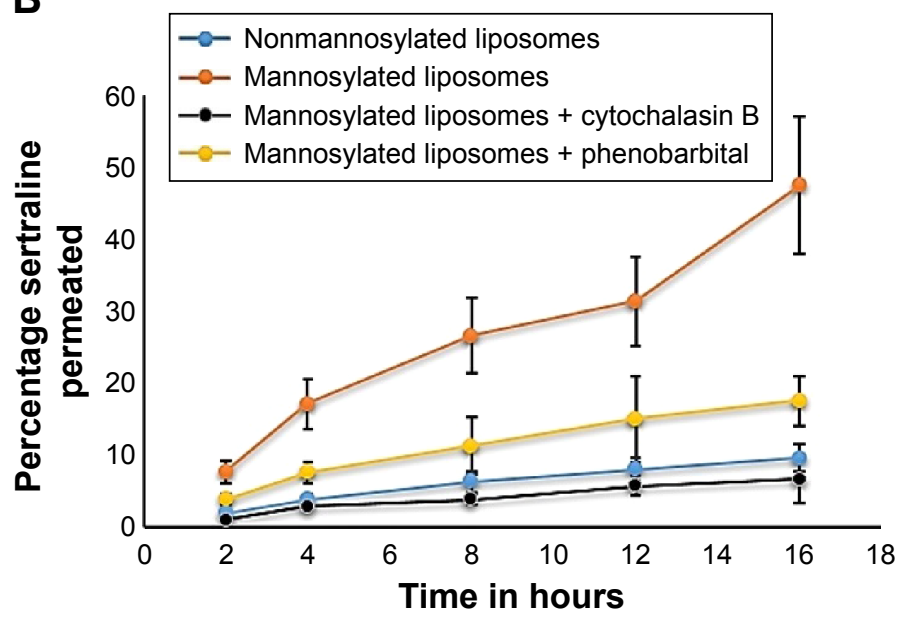

Figure 5 (A) Scanning electron microscopy image of the optimized liposomal formulation; and (B) transendothelial permeability of the optimized sertraline-loaded mannosylated liposomes through an in vitro blood-brain barrier model.

Note: The data represent cumulative percentage sertraline permeated (mean \pm standard deviation, $n=3$ ) at specified time-points.

\section{In vitro $B B B$ transport model}

The endothelialpolyoma middle $\mathrm{T}$ antigen-transformed cell lines were employed as in vitro BBB model to evaluate the transendothelial ability of mannosylated liposomes. Measures of TEER were primarily utilized to assess tight junctions' integrity of monolayers in prior transport studies. The obtained results showed that TEER values before and after the transport study varied between $160 \Omega / \mathrm{cm}^{2}$ and $195 \Omega / \mathrm{cm}^{2}$ to indicate that the monolayer's tightness was robust and controlled. ${ }^{35}$ Figure 5 represents the rank of accumulated percentage sertraline permeated across the in vitro BBB over a period of 16 hours. The percentage of sertraline permeated at 16 hours was $9.5 \% \pm 1.9 \%$ for nonmannosylated sertralineloaded liposomes and $47.5 \% \pm 9.6 \%$ for mannosylated sertraline-loaded liposomes. This significant enhancement $(P<0.05)$ of sertraline permeation would indicate the effectiveness of mannosylation in the transendothelial process of brain-targeting liposomes. In particular, GLUT1 expression at the BBB facilitated this transport across the BBB.

As a confirmation of the transport mechanism, inhibition assay was then performed by preincubation with either cytochalasin B or phenobarbital. ${ }^{1}$ Figure 5 demonstrates that the transendothelial ability of the optimized mannosylated liposomes significantly decreased after this preincubation. Moreover, treatment with cytochalasin B led to a greater reduction of the transendothelial ability than phenobarbital. For example, the percentage of sertraline permeated after 16 hours was $6.7 \% \pm 2.3 \%$ and $17.5 \% \pm 3.5 \%$ for cytochalasin B and phenobarbital preincubation, respectively. This result is attributed to the affinity of cytochalasin B as a ligand to bind to GLUT1, and hence, glucose transport across the endothelial cells was inhibited. ${ }^{36}$ On the other hand, phenobarbital might interact directly with GLUT1 and, hence, abolish the intrinsic activity of the transporter. ${ }^{37}$ In addition, no significant reduction in TEER values was detected in any group during this study to indicate that both the preincubation with the inhibitors and sertraline transport did not disrupt the BBB barrier properties. Regarding the larger size of liposomes compared to mannose moieties, two pathways for liposomal transport might be encountered: a transcytosis pathway that is rapid and of short route and an adsorptive endocytosis pathway that is low and of long route. Similar observations were reported by other researchers that mannosylated liposomes not only could promote the transendothelial penetration but also could be accumulated in the brain with a distinct spatio-temporal pattern. ${ }^{1}$ Moreover, Du et al have proposed a unique study of targeting mannosylated liposomes to the cerebellum and cerebral cortex. ${ }^{38}$ The authors demonstrated that the transcytosis by GLUT1 and GLUT3 was a pathway of mannosylated liposomes into brain, and the special brain distribution of mannosylated liposomes was closely related to the nonhomogeneous distribution of GLUT1 and GLUT3 in the CNS.

\section{Conclusion}

Box-Behnken design was employed to optimize the ultrasound parameters for maximum mannosylation capacity and entrapment of sertraline hydrochloride in its pegylated and mannosylated liposomes as a promising brain-targeting vector. The obtained results showed a modification of vesicular surface by mannosylation with $P$-aminophenyl$\alpha$-D-mannopyranoside. The obtained results showed that 
increasing the sonication temperature resulted in a linear and polynomial decrease in vesicles size with a significant increase in size distribution. However, increasing all sonication parameters resulted in a significant increase in surface charge intensity while decreasing sertraline entrapment and mannosylation capacity. Applying a generalized desirability function, optimized ultrasound parameters of $65^{\circ} \mathrm{C}, 27 \%$, and 59 seconds for ultrasound temperature, amplitude, and time, respectively, were obtained with predicted responses of $81.1 \%, 46.6 \mathrm{~nm}, 0.68,12.3 \mathrm{mV}$, and $77.6 \%$ for $\mathrm{EE}$, vesicular size, skewness, zeta potential, and mannosylation capacity, respectively. Moreover, the transendothelial ability was significantly increased by 2.5 -fold by mannosylation through the intrinsic activity of GLUT1. Despite maximization of the mannosylation capacity of the prepared liposomes, a balance should exist between its transport potential and the recognition by the macrophages to increase its circulation time.

\section{Acknowledgments}

The authors acknowledge Ibrahim Harbi, Sara Abdullah Al-Attas, Razan Hameed Abdullah Alsulami, Noor Tawfiq Omer Sebaih, and Wejdan Abdulrazak Banjar, the students at Faculty of Pharmacy, King Abdulaziz University, Jeddah, $\mathrm{KSA}$, for their assistance in performing the practical part of this study.

\section{Disclosure}

The authors report no conflicts of interest in this work.

\section{References}

1. Hao ZF, Cui YX, Li MH, et al. Liposomes modified with P-aminophenyl-alpha-D-mannopyranoside: a carrier for targeting cerebral functional regions in mice. Eur J Pharm Biopharm. 2013;84(3): $505-516$.

2. Xu X, Warrington AE, Bieber AJ, Rodriguez M. Enhancing CNS repair in neurological disease: challenges arising from neurodegeneration and rewiring of the network. CNS Drugs. 2011;25(7):555-573.

3. Pardridge WM. Blood-brain barrier delivery. Drug Discov Today. 2007; 12(1-2):54-61.

4. Pardridge W. Targeted delivery of protein and gene medicines through the blood-brain barrier. Clin Pharmacol Ther. 2015;97(4):347-361.

5. Chen MY, Hoffer A, Morrison PF, et al. Surface properties, more than size, limiting convective distribution of virus-sized particles and viruses in the central nervous system. $J$ Neurosurg. 2005;103(2): 311-319.

6. Wang T, Zhen Y, Ma X, Wei B, Li S, Wang N. Mannosylated and lipid A-incorporating cationic liposomes constituting microneedle arrays as an effective oral mucosal HBV vaccine applicable in the controlled temperature chain. Colloids Surf B Biointerfaces. 2015;126:520-530.

7. Kong F, Zhou F, Ge L, Liu X, Wang Y. Mannosylated liposomes for targeted gene delivery. Int J Nanomedicine. 2012;7:1079-1089.

8. Zeller K, RahnerWelsch S, Kuschinsky W. Distribution of Glut1 glucose transporters in different brain structures compared to glucose utilization and capillary density of adult rat brains. J Cereb Blood Flow Metab. 1997;17(2):204-209.
9. Vannucci SJ, Maher F, Simpson IA. Glucose transporter proteins in brain: delivery of glucose to neurons and glia. Glia. 1997;1:2-21.

10. Satish PR, Surolia A. Preparation and characterization of glycolipidbearing multilamellar and unilamellar liposomes. Methods Mol Biol. 2002;199:193-202.

11. Duzgunes N. Preparation and quantitation of small unilamellar liposomes and large unilamellar reverse-phase evaporation liposomes. Methods Enzymol. 2003;367:23-27.

12. Silva R, Ferreira H, Little C, Cavaco-Paulo A. Effect of ultrasound parameters for unilamellar liposome preparation. Ultrason Sonochem. 2010;17(3):628-632.

13. Ahmed SE, Martins AM, Husseini GA. The use of ultrasound to release chemotherapeutic drugs from micelles and liposomes. J Drug Target. 2015;23(1):16-42.

14. Basto C, Tzanov T, Cavaco-Paulo A. Combined ultrasound-laccase assisted bleaching of cotton. Ultrason Sonochem. 2007;14(3): 350-354.

15. Pereira-Lachataignerais J, Pons R, Panizza P, Courbin L, Rouch J, Lopez O. Study and formation of vesicle systems with low polydispersity index by ultrasound method. Chem Phys Lipids. 2006;140(1-2): 88-97.

16. Rae J, Ashokkumar M, Eulaerts O, von Sonntag C, Reisse J, Grieser F. Estimation of ultrasound induced cavitation bubble temperatures in aqueous solutions. Ultrason Sonochem. 2005;12(5):325-329.

17. Little C, El-Sharif M, Hepher MJ. The effect of solution level on calorific and dosimetric results in a $70 \mathrm{kHz}$ tower type sonochemical reactor. Ultrason Sonochem. 2007;14(3):375-379.

18. Kilts CD. Potential new drug delivery systems for antidepressants: an overview. J Clin Psychiatry. 2003;64(suppl 18):31-33.

19. Zidan AS, Spinks C, Fortunak J, Habib M, Khan MA. Near-infrared investigations of novel anti-HIV tenofovir liposomes. AAPS J. 2010; 12(2):202-214.

20. Ying X, Wen H, Lu WL, et al. Dual-targeting daunorubicin liposomes improve the therapeutic efficacy of brain glioma in animals. J Control Release. 2010;141(2):183-192.

21. Zidan AS, Mokhtar M. Multivariate optimization of formulation variables influencing flurbiprofen proniosomes characteristics. $J$ Pharm Sci. 2011;100(6):2212-2221.

22. Sarkar S, Das N. Mannosylated liposomal flavonoid in combating agerelated ischemia-reperfusion induced oxidative damage in rat brain. Mech Ageing Dev. 2006;127(4):391-397.

23. Witoonsaridsilp W, Paeratakul O, Panyarachun B, Sarisuta N. Development of mannosylated liposomes using synthesized $\mathrm{N}$-octadecyl-D-mannopyranosylamine to enhance gastrointestinal permeability for protein delivery. AAPS Pharm Sci Tech. 2012;13(2): 699-706.

24. Li SD, Huang L. Stealth nanoparticles: high density but sheddable PEG is a key for tumor targeting. $J$ Control Release. 2010;145(3): $178-181$.

25. Kaur IP, Bhandari R, Bhandari S, Kakkar V. Potential of solid lipid nanoparticles in brain targeting. J Control Release. 2008;127(2): 97-109.

26. Hu K, Li J, Shen Y, et al. Lactoferrin-conjugated PEG-PLA nanoparticles with improved brain delivery: in vitro and in vivo evaluations. $J$ Control Release. 2009;134(1):55-61.

27. Fromherz P, Ruppel D. Lipid vesicle formation: the transition from open disks to closed shells. FEBS Lett. 1985;179(1):155-159.

28. Ikonen M, Murtomaki L, Kontturi K. Microcalorimetric and zeta potential study on binding of drugs on liposomes. Colloids Surf B Biointerfaces. 2010;78(2):275-282.

29. Makino K, Yamada T, Kimura M, Oka T, Ohshima H, Kondo T. Temperature- and ionic strength-induced conformational changes in the lipid head group region of liposomes as suggested by zeta potential data. Biophys Chem. 1991;41(2):175-183.

30. Kamal N, Cutie AJ, Habib MJ, Zidan AS. QbD approach to investigate product and process variabilities for brain targeting liposomes. J Liposome Res. Epub 2014 Oct 13. 
31. Isailović BD, Kostić IT, Zvonar A, et al. Resveratrol loaded liposomes produced by different techniques. Innov Food Sci Emerg Technol. 2013; 19(0):181-189.

32. Caddeo C, Teskac K, Sinico C, Kristl J. Effect of resveratrol incorporated in liposomes on proliferation and UV-B protection of cells. Int J Pharm. 2008;363(1-2):183-191.

33. Ninomiya K, Yamashita T, Kawabata S, Shimizu N. Targeted and ultrasound-triggered drug delivery using liposomes co-modified with cancer cell-targeting aptamers and a thermosensitive polymer. Ultrason Sonochem. 2014;21(4):1482-1488.

34. Yu W, Zhang N, Li C. Saccharide modified pharmaceutical nanocarriers for targeted drug and gene delivery. Curr Pharm Des. 2009;15(32): 3826-3836.

35. Koto T, Takubo K, Ishida S, et al. Hypoxia disrupts the barrier function of neural blood vessels through changes in the expression of claudin-5 in endothelial cells. Am J Pathol. 2007;170(4):1389-1397.
36. Dick AP, Harik SI, Klip A, Walker DM. Identification and characterization of the glucose transporter of the blood-brain barrier by cytochalasin B binding and immunological reactivity. Proc Natl Acad Sci US A. 1984; 81(22):7233-7237.

37. Klepper J, Fischbarg J, Vera JC, Wang D, De Vivo DC. GLUT1-deficiency: barbiturates potentiate haploinsufficiency in vitro. Pediatr Res. 1999;46(6):677-683.

38. Du D, Chang N, Sun S, et al. The role of glucose transporters in the distribution of p-aminophenyl-alpha-d-mannopyranoside modified liposomes within mice brain. J Control Release. 2014;182:99-110.

\section{Publish your work in this journal}

Drug Design, Development and Therapy is an international, peerreviewed open-access journal that spans the spectrum of drug design and development through to clinical applications. Clinical outcomes, patient safety, and programs for the development and effective, safe, and sustained use of medicines are a feature of the journal, which has also been accepted for indexing on PubMed Central. The manuscript management system is completely online and includes a very quick and fair peer-review system, which is all easy to use. Visit http://www.dovepress.com/testimonials.php to read real quotes from published authors.

Submit your manuscript here: http://www.dovepress.com/drug-design-development-and-therapy-journal 